\title{
ASPECTOS DOS EFEITOS DO SONO NO APARELHO DIGESTÓRIO
}

\author{
Roberto Oliveira DANTAS e Cynthia Gutierrez ABEN-ATHAR
}

RESUMO - Racional - Durante o sono ocorrem modificações no funcionamento do aparelho digestório decorrentes da diminuição da influência do sistema nervoso central. Embora haja resultados controversos, decorrentes das dificuldades metodológicas de estudo do aparelho digestório durante o sono, no geral, a atividade está diminuída. Objetivos - Descrever o que é conhecido sobre o efeito do sono nos diferentes órgãos do aparelho digestório. Durante o sono ocorrem: diminuição da salivação, da freqüência de deglutições, da pressão do esfíncter superior do esôfago e do número de contrações primárias do esôfago. Dados de fontes de referências - Alguns trabalhos demonstraram esvaziamento gástrico lento, outros indicam que durante o sono REM o esvaziamento é rápido. O ritmo da atividade motora intestinal é mais regular à noite do que durante o dia. Durante o sono a fase II do complexo motor migrante intestinal está bastante diminuída, podendo estar ausente. A velocidade de propagação da fase III está diminuída, bem como o índice de motilidade. Situação semelhante é observada no cólon, que tem o tônus e as contrações diminuídas. A pressão do canal anal diminui durante o sono e a atividade do reto está aumentada. Entretanto, a pressão do canal anal continua maior do que a do reto e as contrações no reto são, em sua maioria, retrógradas. Síntese dos dados - O mais freqüente mecanismo causador do refluxo gastroesofágico é o relaxamento transitório do esfíncter inferior do esôfago. A freqüência deste relaxamento está diminuída durante o sono, o que explica a menor ocorrência de episódios de refluxo durante a noite. Neste período o principal causador de refluxo é a pressão baixa do esfíncter. $\mathrm{O}$ refluxo nestas condições é mais grave porque o paciente geralmente está na posição supina, portanto a gravidade não contribui para a retirada do material refluído do esôfago, a salivação está diminuída, dificultando a neutralização do ácido, e as contrações primárias do esôfago também estão diminuídas. Conclusões - Considerando estes fatores é esperado que episódio de refluxo ocorrido durante o sono tenha maior duração do que aquele ocorrido no estado de vigília. É demonstrado que o decúbito lateral direito favorece episódios de refluxo. É conhecido também a possibilidade do refluxo gastroesofágico estar associado a broncoespasmo noturno e apnéia do sono.

DESCRITORES - Sono. Sistema digestório. Fisiologia digestória. Refluxo gastroesofágico.

A função do aparelho digestório sofre influência do meio ambiente e de estímulos recebidos pelo ser humano. O estado de sono ou vigília faz com que a influência do meio externo seja melhor ou pior percebida.

São identificados no sono dois estados comportamentais: o sono sincronizado ou não-REM (do inglês "rapid eye movement") e o sono dessincronizado ou REM. O sono nãoREM é dividido em estágios de 1 a 4 , que representam progressivamente a profundidade do sono. Nestes estágios ocorre diminuição da atividade neuromuscular com ausência de sonhos. O sono não-REM caracteriza-se por atividade elétrica cerebral síncrona com elementos próprios como fusos de sono, complexos $\mathrm{K}$ e ondas lentas de grande amplitude. Ocorre também diminuição da atividade do sistema nervoso autônomo simpático e um aumento do tônus parassimpático, a níveis mais altos do que durante a vigília. A atividade muscular diminui, com redução do tônus muscular, e a atividade mental atinge o seu mínimo. Há relativa inatividade do cérebro. O sono REM não é dividido em estágios e caracteriza-se pela dessincronização eletroencefalográfica, episódios de movimentos oculares rápidos, movimentos da musculatura do ouvido médio, breves abalos musculares de membros e atonia muscular, tornando a

Divisão de Gastroenterologia do Departamento de Clínica Médica da Faculdade de Medicina de Ribeirão Preto da Universidade de São Paulo

Endereço para correspondência: Dr. Roberto Oliveira Dantas - Departamento de Clínica Médica - Faculdade de Medicina de Ribeirão Preto - USP - 14049-900 - Ribeirão Preto, SP. 
musculatura esquelética paralisada. Ocorre instabilidade do sistema nervoso autônomo simpático. O tônus do sistema nervoso autônomo parassimpático é o mesmo do sono não-REM. No sono REM ocorrem sonhos, o que indica atividade mental. Considera-se que no sono REM o cérebro está ativado em um corpo paralisado ${ }^{(1)}$.

O estudo do efeito de sono no aparelho digestório tem limitações decorrentes do fato da maioria dos métodos de estudo serem invasivos e dificultarem o sono do indivíduo, necessitarem de sua colaboração quanto a movimentos e precisarem de posições que permitam sua avaliação contínua. Apesar destas limitações, grande número de estudos têm sido desenvolvidos. De modo geral, o sono provoca diminuição da atividade do aparelho digestório. Nos estudos realizados, é freqüente encontrarem-se resultados contraditórios; entretanto, com o conjunto dos trabalhos, algumas conclusões puderam ser atingidas.

Durante o sono ocorre diminuição da salivação e da frequiência de deglutição. O fluxo de saliva é inibido durante o sono ${ }^{(36)}$, o que é importante porque prejudica a neutralização do ácido intra-esofágico, quando ocorre refluxo gastroesofágico. Associada à diminuição da secreção salivar, ocorre redução na freqüência de deglutição ${ }^{(3,17,23,28)}$. Em oito pessoas normais foi observada a média e desvio padrão de 1,61 \pm 0,87 deglutições por minuto durante o período de vigília, de $0,24 \pm 0,25$ no sono em estágio 1 e de $0,06 \pm 0,04$ no sono em estágios de 2 a $5^{(17)}$.

A pressão do esfíncter superior do esôfago (ESE) cai acentuadamente quando a pessoa dorme ${ }^{(17)}$. O principal componente do ESE é o músculo cricofaríngeo. Como se trata de músculo estriado, é necessário constante ação dos nervos, modulada por diferentes estímulos para a manutenção do tônus. Durante o sono o estímulo nervoso diminui e o tônus muscular praticamente desaparece. A pressão registrada neste momento representa a pressão exercida pelos órgãos vizinhos, ou seja, a posição anatômica da transição faringoesofágica faz com que mesmo sem o tônus do músculo cricofaríngeo, ocorram pressões em torno de $8 \mathrm{~mm} \mathrm{Hg}^{(17)}$.

O corpo do esôfago é menos afetado pelo sono. Como ocorre menor número de deglutições, também ocorre menor número de contrações primárias. A associação entre diminuição do volume de saliva e menor número de contrações primárias é particularmente importante quando ocorre refluxo gastroesofágico, situação em que a retirada e neutralização do material refluído está prejudicada.

Durante o sono o esôfago demora a perceber a presença do refluxo ácido e quase sempre a resposta ao mesmo é precedida pelo despertar. As contrações secundárias, que são contrações espontâneas desencadeadas pela distensão do esôfago, também estão diminuídas nos estágios 1 a 4 do sono, a exemplo do que ocorre com as contrações primárias desencadeadas pela deglutição. Entretanto, há diferenças durante o sono REM, quando se observa que as contrações secundárias têm a mesma freqüência encontrada na pessoa acordada ${ }^{(33)}$.

A secreção gástrica tem ritmo circadiano, com a maior produção de secreção ácida ocorrendo entre 22 horas e 2 horas ${ }^{(25)}$. Portanto, existe aumento da secreção gástrica no período noturno, independente da pessoa estar dormindo ou não. Os estudos realizados até o momento não conseguiram demonstrar que o sono influi na secreção gástrica ${ }^{(33)}$. Seu aumento no período noturno é mais acentuado no paciente com úlcera duodenal, o que tem implicações terapêuticas ${ }^{(32)}$.

Quanto ao esvaziamento gástrico, há estudos que demonstram ser rápido durante o sono REM e lento ao despertar. Outros demonstraram esvaziamento gástrico lento, sem diferenças entre o sono REM e não-REM ${ }^{(33)}$. As diferenças observadas poderiam ser conseqüência de ritmo circadiano e não do sono. Com sólidos foi demonstrado que o esvaziamento gástrico à noite é mais lento do que pela manhã $\tilde{a}^{(9)}$. Em relação ao ritmo elétrico de base (BER) gástrico, foi observado que o sono não-REM é associado com alterações ou desestabilização do BER, o que sugere que a pessoa acordada consegue estabilizar melhor, via sistema nervoso central, o funcionamento gástrico e o esvaziamento gástrico normal ${ }^{(33)}$. De modo geral, deve-se considerar que a função motora gástrica diminui durante o sono, o que pode ser conseqüência de ritmo circadiano e não efeito do sono.

A motilidade intestinal caracteriza-se por padrão interdigestivo e outro pós-prandial. O padrão interdigestivo é representado por atividade motora periódica, conhecida como complexo motor migrante (CMM) ou complexo motor interdigestivo (CMI), que se caracteriza por três fases: fase I de total ausência de contrações, fase II de contrações irregulares e fase III de contrações repetidas com freqüência de 9 a 12 por minuto. Essas contrações se iniciam no estômago e se propagam até o fim do intestino delgado. A periodicidade com que ocorre este complexo motor varia de 90 a 120 minutos $^{(21,22)}$. Este padrão interdigestivo é interrompido pela ingestão de alimentos, que produz intensa atividade motora, irregular com duração de 3 a 4 horas ${ }^{(21,22)}$. O ritmo de atividade motora intestinal é mais regular durante a noite do que durante o dia. $\mathrm{O}$ comprimento do ciclo interdigestivo, ou seja, o tempo em que se completa, é menor durante o sono do que acordado.

Durante o sono, a fase II do CMM tem duração bastante diminuída, podendo estar ausente. Esta duração, que é de $123 \pm 62$ minutos (média \pm EPM) quando a pessoa está acordada, cai para $3 \pm 6$ minutos durante o sono. Ocorre também diminuição da velocidade da fase $\mathrm{III}^{(21,22)}$. Avaliando-se o índice de motilidade, observou-se que ele foi menor durante todos os estágios do sono, quando comparado com o período de vigília. $\mathrm{O}$ índice de motilidade foi de 4,5 $\pm 0,4$ com o paciente acordado, 3,2 \pm 0,3 nos estágios 1 e 2 do sono, 2,3 $\pm 0,3$ nos estágios 3 e 4 e 3,3 \pm 0,4 no sono REM. Não houve diferença entre os resultados das diferentes fases do sono ${ }^{(11)}$.

A ingestão de alimentos modifica a motilidade, interrompendo o CMM e provocando contrações repetitivas e intensas. Quando a pessoa dorme após a refeição, a atividade do intestino diminui e o padrão motor interdigestivo retorna mais precocemente, ou seja, a duração dos movimentos do intestino, característicos do período pósprandial, está diminuída ${ }^{(21,22)}$. Nesta situação, a fase II do CMM tem 
duração maior do que aquela observada durante o sono isoladamente. A hipótese para explicar esta observação é a diminuição do tônus vagal no intestino durante o sono.

É importante considerar que a motilidade intestinal pode afetar o sono. É conhecido que no período pós-prandial algumas pessoas têm sonolência. Em 1920 foi observado que a distensão de balão intrajejunal provoca sono ${ }^{(2)}$. Outros estudos realizados em humanos e em animais de experimentação confirmaram a relação entre distensão do intestino e o sono $^{(33)}$. O mecanismo que explica esta relação ainda não é completamente conhecido, mas há possibilidades de que seja conseqüência da liberação de hormônios gastrointestinais, como a colecistocinina, ou de estimulação do sistema nervoso central pela distensão do intestino ${ }^{(33)}$.

Vários trabalhos têm demonstrado que a atividade do cólon está diminuída durante o sono, o que inclui o cólon transverso, descendente e sigmóide, com aumento da atividade ao acordar ${ }^{(8,26)}$. Esta diminuição inclui o tônus e as contrações. O aumento da atividade ao despertar é observado quando este ocorre espontaneamente, e difere de quando a pessoa acorda abruptamente. Com o despertar espontâneo, ocorrem no cólon contrações peristálticas de grande amplitude, e no despertar abrupto as contrações são segmentares ${ }^{(33)}$.

A pressão do canal anal está diminuída durante o sono. Ao mesmo tempo, a atividade motora do reto está aumentada. As condições que mantém a continência fecal durante o sono são de natureza a combinar fatores que impedem a perda fecal. Embora a pressão no canal anal esteja diminuída, ela é sempre maior do que a registrada no reto e as contrações retais são, em sua maioria, retrógradas ${ }^{(33)}$.

Com a pessoa acordada, a distensão retal com balão provoca aumento do tônus do esfíncter anal externo e relaxamento do esfíncter anal interno. Durante o sono, a resposta do esfíncter anal interno está inalterada e a resposta do esfíncter anal externo está diminuída. Estas condições favorecem o escape fecal, que não ocorre em função dos mecanismos citados, ou seja, contrações retrógradas no reto e pressão no canal anal maior do que a pressão no reto. Se ocorre comprometimento destes mecanismos protetores, a incontinência fecal pode ocorrer, o que acontece no diabetes e naqueles com anastomose íleo-anal.

O sistema nervoso entérico é o grande controlador das funções do aparelho digestório. O sistema nervoso central funciona como receptor das informações vindas dos diferentes órgãos, captados nos neurônios de sensibilidade, e modulador destas funções. Durante o sono as funções do aparelho digestório persistem, entretanto há modificação da modulação exercida pelo sistema nervoso central ${ }^{(40)}$.

\section{REFLUXO GASTROESOFÁGICO DURANTE O SONO}

A doença do refluxo gastroesofágico (DRGE) compreende qualquer condição clínica sintomática, com ou sem evidências de lesão tecidual esofágica, secundárias a refluxo do conteúdo gástrico para o esôfago. Apesar de ter alta prevalência na população geral, somente em torno de 50\% dos indivíduos com DRGE apresentam lesões esofágicas e suas complicações ${ }^{(37)}$. A progressão do refluxo fisiológico para a lesão da mucosa esofágica depende de fatores anatômicos e fisiológicos associados, principalmente, à integridade estrutural e funcional do esfíncter inferior do esôfago (EIE) e à depuração ácida esofágica. As apresentações mais graves da doença parecem estar relacionadas a episódios recurrentes de refluxo durante o decúbito e o sono.

JOHNSON e DeMEESTER ${ }^{(15)}$ e DeMEESTER et al. ${ }^{(6)}$, utilizandose de manometria e pHmetria de 24 horas, observaram que existem padrões distintos de refluxo gastroesofágico (RGE) durante o sono e no paciente desperto. O refluxo na posição ortostática ocorre mais freqüentemente no período pós-prandial, associado aos relaxamentos transitórios do EIE de menor gravidade. Usualmente consiste de 2 ou 3 episódios de refluxo, rapidamente depurados. O refluxo na posição supina está usualmente associado ao sono, com aumento do tempo de depuração ácida esofágica e da exposição ácida noturna. Há evidências de que o mecanismo dominante de refluxo possa variar em função da gravidade da doença, com os relaxamentos transitórios sendo predominantes na doença de menor gravidade ${ }^{(7)}$ e os mecanismos associados à hérnia hiatal e EIE hipotensivo na doença mais grave. Os relaxamentos transitórios do EIE estão associados à distensão gástrica pelas refeições, sendo praticamente ausentes durante o sono e a anestesia.

A depuração ácida do esôfago, refletida pela duração de tempo em que a mucosa esofágica permanece com $\mathrm{pH}<4$ após um episódio de refluxo, requer tanto esvaziamento esofágico, quanto salivação efetivas. A depuração ácida normal é realizada em duas etapas: primeiramente, ocorre a eliminação do volume do material refluído e na segunda etapa o bicarbonato da saliva deglutida tampona a acidez que restou do refluxo. Em indivíduos normais, a resposta inicial ao refluxo ácido é usualmente o peristaltismo primário, provavelmente devido à alta taxa de deglutições no estado desperto e pelo estímulo à deglutição pelo refluxo ácido. $\mathrm{O}$ peristaltismo secundário é menos comum, tendo papel importante durante o decúbito e o sono, provavelmente pela supressão da salivação e deglutição durante o sono ${ }^{(12)}$. As deglutições de saliva provocam menor amplitude de contração do que as deglutições com algum volume de líquido ou sólido ${ }^{(34)}$, portanto com maior possibilidade de serem ineficazes. Normalmente são necessárias 8 a 10 deglutições para que o $\mathrm{pH}$ esofágico se eleve acima de 4. Durante o sono a freqüência de deglutições é de aproximadamente 6 por hora e ocorrem em associação com movimentos de despertar, principalmente nos estágios 1 e 2 do sono.

ORR et al. ${ }^{(31)}$, comparando os resultados da pHmetria de 24 horas em pacientes com e sem esofagite erosiva à endoscopia, demonstraram que o tempo de exposição ácida total e o número de episódios de refluxo que requerem mais de 5 minutos para serem depurados, que ocorrem mais freqüentemente durante o sono, são os fatores mais fidedignos de discriminação entre os dois grupos. Com o intuito de se avaliar a depuração ácida do esôfago durante os diferentes estágios do sono, os mesmos autores ${ }^{(27,28)}$ utilizaram-se de modelo de infusão de 
ácido $(\mathrm{HCl} 0,1 \mathrm{~N})$ no esôfago distal em períodos de sono REM e nãoREM documentados por meio de polissonografia. Observou-se que a depuração ácida esofágica estava invariavelmente associada com episódios de despertar e, se o despertar não ocorresse, havia prolongamento acentuado no tempo de exposição ácida do esôfago.

Os pacientes portadores de DRGE podem, portanto, ser divididos de acordo com sua forma de apresentação em refluidores diurnos, refluidores noturnos e refluidores mistos. A elevação da cabeceira da cama durante o sono produz melhora significativa da depuração ácida esofágica nos refluidores noturnos, apesar de não alterar a freqüência dos episódios de refluxo ${ }^{(16)}$. O decúbito lateral direito, por sua vez, está associado com maior percentual de tempo com $\mathrm{pH}$ esofágico abaixo de 4 e aumento do tempo de depuração ácida do esôfago. Portanto, em indivíduos com RGE noturno, deve-se dar preferência ao decúbito lateral esquerdo ${ }^{(19)}$. Além disso, o uso de benzodiazepínicos e álcool antes de dormir têm sido implicados como fatores de prolongamento da depuração esofágica, podendo tornar episódios benignos de RGE em eventos nocivos à mucosa esofágica ${ }^{(29,38)}$.

O broncoespasmo noturno em asmáticos e a tosse crônica noturna são sintomas pulmonares que parecem estar relacionados à ocorrência de $\mathrm{RGE}^{(20)}$. A despeito dos vários estudos nesse assunto, ainda há controvérsias sobre a validade dessa associação. É sabido que a resposta do esôfago a estímulos nocivos, incluindo o ácido, está amplificada durante o sono, a fim de proteger a árvore traqueobrônquica da aspiração do conteúdo esofágico. Há maior eficiência dos mecanismos de despertar e redução do tempo de latência à deglutição, o que é mais evidente com maiores volumes e menores valores de $\mathrm{pH}$ do material refluído ${ }^{(30)}$. Dessa forma, a aspiração propriamente dita do conteúdo gástrico é rara, apesar de haver estudos demonstrando, após a injeção de material marcado radioativamente no estômago, a presença de radioatividade pulmonar na manhã seguinte ${ }^{(4)}$. MANSFIELD e $\operatorname{STEIN}^{(24)}$ relataram que a presença de ácido no esôfago distal, por si só, é capaz de aumentar a resistência pulmonar e reduzir o fluxo aéreo em pacientes com teste de perfusão ácida positivo. O alívio dos sintomas respiratórios com o uso de antiácidos é outro fator que colabora como evidência desta associação ${ }^{(10)}$. O fato dos sintomas serem desencadeados principalmente durante a noite pode estar relacionado a maior duração dos refluxos noturnos e ao déficit de depuração ácida esofágica mais ineficiente durante o decúbito e o sono. DAVID et al. ${ }^{(5)}$ demonstraram que $47 \%$ dos pacientes com bronquite crônica têm RGE e que o principal fator predisponente nestes pacientes era o déficit de depuração esofágica

Vários estudos têm descrito alta incidência de RGE em pacientes portadores de apnéia do sono ${ }^{(18,35)}$. Há várias possíveis razões para a associação entre estas síndromes, já que possuem fatores predisponentes em comum, como a obesidade e uso de álcool. Além disso, o RGE pode precipitar sintomas sugestivos de apnéia por causar inflamação e edema da laringe, podendo piorar a obstrução das vias aéreas. A apnéia obstrutiva, por sua vez, tem o potencial de desencadear episódios de refluxo, pois está associada a esforço inspiratório contínuo contra uma via aérea superior fechada, levando à redução da pressão intratorácica e aumento da pressão transdiafragmática. A redução da eficiência do sono poderia predispor ainda a relaxamentos transitórios do EIE e promoção de refluxos ácidos. Todavia, a associação direta entre a apnéia obstrutiva e RGE ainda não foi bem identificada, não havendo relação temporal clara entre os episódios de apnéia e os refluxos. Em estudo realizado por ING et al. ${ }^{(13)}$, observou-se que o RGE pode contribuir para a patogênese do despertar, que é reduzido significativamente pelo uso de bloqueadores $\mathrm{H}_{2}$ (nizatidine), sem melhora, porém, do índice apnéia-hipopnéia. $\mathrm{O}$ uso de pressão positiva em vias aéreas (CPAP) via nasal reduz os parâmetros de RGE medidos pela pHmetria de 24 horas tanto nos pacientes com, como sem apnéia obstrutiva, tendo, portanto, efeito inespecífico, possivelmente por diminuir a pressão transdiafragmática. Estudos maiores devem ser realizados para melhor investigar a correlação entre estas duas doenças. Em crianças, alguns estudos sugerem associação entre RGE, apnéia noturna e morte súbita, mas a maioria dos estudos bem controlados nessa área não encontraram relação consistente entre estes fenômenos ${ }^{(14,39)}$.

Nos últimos anos têm sido desenvolvidos estudos sobre o sono, importante período de recuperação de atividades fisiológicas. No geral, durante o sono o aparelho digestório diminui sua atividade. O conhecimento das alterações fisiológicas que ocorrem nesta fase melhora a compreensão de alguns sintomas que podem ocorrer no período noturno, como a incontinência fecal e a tosse noturna, e a razão dos episódios de refluxo gastroesofágico serem menos freqüentes durante o sono.

Dantas RO, Aben-Athar CG. Effects of sleep on the gastrointestinal tract. Arq Gastroenterol 2002;39(1):55-59.

ABSTRACT - Background - During sleep the gastrointestinal system function is markedly reduced most of the time. Objectives - In this revision we described the effect of sleep on the digestive system. Salivation, swallowing rate, upper esophageal sphincter pressure and number of primary esophageal contractions have all been shown to be reduced during sleep. Data sources - Gastric emptying is slow during sleep but the REM sleep is associated with faster gastric emptying. During the night we have a more regular intestinal motility than during the day. During sleep, phase II of the migrating motor complex cycle is virtually absent, both during diurnal or nocturnal sleep. The nocturnal velocity of migrating motor complex propagation in the proximal small bowel is slower than the diurnal velocity. The colon has a decrease in tonus and contractions. The anal canal pressure is lower and rectum activity is higher during sleep than during the awake state, but the anal pressure is still higher than the 
rectum pressure and the rectum contractions are most frequently retrograde. Data synthesis - Transient lower esophageal sphincter relaxation is the most frequent cause of gastroesophageal reflux. The frequency of this transient relaxation is very low during sleep. Gastroesophageal reflux during sleep is more frequently associated with a low lower esophageal sphincter pressure. Conclusions - In this situation the disease is worse because the patient is in the supine position, so gravity does not help the acid esophageal clearance, salivation is decreased and the primary esophageal contraction is not frequent, a fact causing a prolongation of acid clearance during sleep. The right lateral position during sleep causes more reflux episodes than the left lateral position. The gastroesophageal reflux may be associated with nocturnal wheezing, chronic nocturnal cough and sleep apnea.

HEADINGS - Sleep. Digestive system. Digestive physiology. Gastroesophageal reflux.

\section{REFERÊNCIAS BIBLIOGRÁFICAS}

1. Alóe F, Silva AB. Sono normal e polisonografia. In: Pinto JA, editor. Ronco e apnéia do sono. Rio de Janeiro: Revinter; 2000. p. 9-16.

2. Alvarez WC. Physiologic studies on the motor activities of the stomach and bowel in man. Am J Physiol 1920;88:658-60.

3. Castiglione F, Emde C, Armstrong D, Schneider C, Banerfeind P, Stacher G, Blum AL. Nocturnal oesophageal motor activity is dependent on sleep stage. Gut 1993;34:1653-9.

4. Chernow B, Johnson LF, Janowitz WR, Castell DO. Pulmonary aspiration as a consequence of gastroesophageal reflux: a diagnostic approach. Dig Dis Sci 1979;24:839-44.

5. David P, Denis P, Nouvet CG, Pasquis P, Lefrancois R, Morere P. Lung function and gastroesophageal reflux during chronic bronchitis. Bull Eur Physiopathol Respir 1982;18:81-6.

6. DeMeester TR, Johnson LF, Joseph GJ, Toscano MS, Hall AW, Skinner DB. Patterns of gastroesophageal reflux in health and disease. Ann Surg 1976;184:459-70

7. Dent J, Dodds WJ, Friedman RH, Sekiguchi T, Hogan WJ, Arndorfer RC, Petrie DJ. Mechanism of gastroesophageal reflux in recumbent asymptomatic human subjects. J Clin Invest 1980;65:256-67.

8. Furukawa Y, Cook IJ, Panagopoulos V, McEvoy RD, Sharp DJ, Simula M. Relationship between sleep patterns and human colonic motor patterns. Gastroenterology 1994;107:1372-81.

9. Goo RH, Moore JG, Greenburg E, Alazraki NP. Circadian variation in gastric emptying of meals in humans. Gastroenterology 1987;93:515-8.

10. Goodall RJR, Earis JE, Cooper DN, Bernstein A, Temple JG. Relationship between asthma and gastro-oesophageal reflux. Thorax 1981;36:116-21.

11. Gorard DA, Vesselinova-Jenkins CK, Libby GW, Farthing MJG. Migrating motor complex and sleep in health and irritable bowel syndrome. Dig Dis Sci 1995;40:2383-9.

12. Holloway RH. Esophageal body motor response to reflux events: secondary peristalsis. Am J Med 2000;108(4A):20-6.

13. Ing AJ, Ngu MC, Dreslin ABX. The role of esophageal reflux in the pathogenesis of obstructive sleep apnea. Am J Respir Crit Care Med 1995;151:A102.

14. Jeffery HE, Reid I, Rahilly P, Read DJ. Gastroesophageal reflux in "near miss" sudden infant death in active but not quiet sleep. Sleep 1980;3:393-9.

15. Johnson LF, DeMeester TR. Twenty-four hour $\mathrm{pH}$ monitoring of the distal esophagus. Am J Gastroenterol 1974;62:325-32.

16. Johnson LF, DeMeester TR. Evaluation of the head of the bed, bethanecol and antacid foam tablets on gastroesophageal reflux. Dig Dis Sci 1981;26:67380 .

17. Kahrilas PJ, Dodds WJ, Dent J, Haeberle B, Hogan WJ, Arndorfer RC. Effect of sleep, spontaneous gastroesophageal reflux, and a meal on upper esophageal sphincter pressure in normal human volunteers. Gastroenterology 1987;92:446-71.

18. Kerr P, Shoenut JP, Millar T, Buckle P, Kryger MH. Nasal CPAP reduces gastroesophageal reflux in obstructive sleep apnea syndrome. Chest 1992;101:1539-44.

19. Khoury RM, Camacho-Lobato L, Katz PO, Mohiuddin MA, Castell DO. Influence of spontaneous sleep positions on nightime recumbent reflux in patients with gastroesphageal reflux disease. Am J Gastroenterol 1999;94:2069-73.
20. Kiljander TO, Salomaa ER, Hietanen EK. Gastroesophageal reflux in asthmatics: a double-blind, placebo-controlled crossover study with omeprazole. Chest 1999; 116:1257-64.

21. Kumar D, Idzikowski, Wingate DL, Soffer EE, Thompson P, Siderfin C. Relationship between enteric migrating motor complex and the sleep cycle. Am J Physiol 1990;259:G983-90.

22. Kumar D. Modulation of motor activity by sleep. In: Kumar D, Wingate D, editors An ilustrated guide to gastrointestinal motility. 2.ed. Edinburg: Churchill Livingstone; 1993. p. 95-103.

23. Lear CSC, Flanagan JB Jr, Moorees CFA. The frequency of deglutition in man Arch Oral Biol 1965;10:83-96.

24. Mansfield LE, Stein MR. Gastroesophageal reflux and asthma: a possible reflex mechanism. Ann Allergy 1978;41:224-6.

25. Moore JG, Enghert E. Circadian rhythm of gastric acid secretion in man. Nature 1970;226:1261-2.

26. Narducci F, Bassotti G, Gaburri M, Morelli A. Twenty four hour manometric recording of colonic motor activity in healthy man. Gut 1987;28:17-25.

27. Orr WC, Robinson MG, Johnson LF. Acid clearing during sleep in the pathogenesis of reflux esophagitis. Dig Dis Sci 1981:26:423-7.

28. Orr WC, Johnson LF, Robinson MG. The effect of sleep on swallowing, esophageal peristalsis, and acid clearance. Gastroenterology 1984;86:814-9.

29. Orr WC, Robinson MG, Rundell OH. The effect of hypnotic drugs on acid clearance during sleep [abstract]. Gastroenterology 1985;88:1526.

30. Orr WC, Robinson MG, Johnson LF. The effect of acid volume on arousals from sleep and acid clearance. Chest 1991;99:351-5.

31. Orr WC, Allen ML, Robinson MG. The pattern of nocturnal and diurnal esophageal acid exposure in the pathogeneis of erosive mucosal damage. Am J Gastroenterol 1994;89:509-12.

32. Orr WC. Gastrointestinal disorders. In: Kryger MH, Roth T, Dement WC, editors. Principles and practice of sleep medicine. 3.ed. Philadelphia: WB Saunders: 2000. p. 1113-22.

33. Orr WC. Gastrointestinal physiology. In: Kryger MH, Roth T, Dement WC, editors. Principles and practice of sleep medicine. 3.ed. Philadelphia: WB Saunders: 2000. p. 279-88

34. Richter JE, Wu WC, Johns DN, Blackwell JN, Nelson JL, Castell JA, Castell DO. Esophageal manometry in 95 healthy adult volunteers. Dig Dis Sci 1987;32:583-92.

35. Samelson CF. Gastroesophageal reflux and obstructive sleep apnea. Sleep 1989; $12: 475-6$

36. Schneyer LH, Pigman W, Hanahan L, Gilmore RW. Rate of flow of human parotid, sublingual, and submaxillary secretions during sleep. J Dent Res 1956;35:109-14

37. Tibbling L. Epidemiology of gastro-oesophageal reflux disease. Scand J Gastroenterol 1984;19 Suppl 106:14-5.

38. Vitale GC, Cheadle WG, Pake IB, Sadek AS, Michel ME, Cuschierri A. The effect of alcohol on nocturnal gastroesophageal reflux. JAMA 1987;258:2077-9.

39. Walsh JK, Farrell MK, Keenan WJ, Lucas M, Kramer M. Gastroesophageal reflux in infants: relation to apnea. J Pediatr 1981;99:197-201.

40. Wood JD, Alpers DH, Andrews PLR. Fundamentals of neurogastroenterology. Gut 1999;45 Suppl 2:6-16.

Recebido em 19/4/2001 Aprovado em 14/8/2001 